\title{
Actuación de las Fuerzas de Seguridad durante aislamiento social, preventivo y obligatorio en la Provincia de Córdoba
}

\author{
Action of the Security Forces during social, preventive and mandatory \\ isolation in the Province of Córdoba \\ Valeria Plaza Schaefer ${ }^{1}$ \\ Delia M. Sanchez ${ }^{2}$
}

\section{Resumen}

Desde el Programa de Extensión "Seguridad y Derechos Humanos" ${ }^{3}$ de la Facultad de Ciencias Sociales (FCS) de la Universidad Nacional de Córdoba (UNC) realizamos un relevamiento de casos sobre prácticas abusivas por parte de las fuerzas de seguridad en el marco de las detenciones y controles establecidos para garantizar el aislamiento social y obligatorio en Provincia de Córdoba.

Nos propusimos comprender las condiciones, situaciones y actores que habilitan las mismas; como así también generar instancias de difusión con organizaciones sociales y la comunidad universitaria, y de articulación con los actores estatales para aportar al diseño de políticas preventivas.

En el presente trabajo caracterizamos, por un lado, el proceso de registro realizado en el primer momento de la pandemia: las acciones y estrategias de visibilización y articulaciones emprendidas. Y, por otro lado, planteamos algunas cuestiones conceptuales y metodológicas en relación a las estrategias de trabajo conjunto con

Recibido: 30 de septiembre de 2021 Aceptado: 20 de diciembre de $2021 \sim$ Publicado: 7 de enero de 2022

${ }^{1}$ Abogada FD UNC y Doctora en Ciencias Sociales UBA. Investigadora asistente CIECS CONICET. Docente "El derecho y la constitución de las instituciones", "Derecho Constitucional” y "Derechos Humanos" de la FCS UNC, Córdoba, Argentina. Correo electrónico: valeria.plaza@unc.edu.ar ID http://orcid.org/0000-00019396-3874

${ }^{2}$ Licenciada en Trabajo Social. Egresada UNLP. Maestranda en Trabajo Social con Mención en intervención social, UNC. Se desempeña profesionalmente en la Secretaría de Niñez, Adolescencia y Familia en la Provincia de Córdoba. Docente Adscripta e Investigadora en FCS-UNC, Argentina. Correo Electrónico: delia.sanchez@mi.unc.edu.ar ID http://orcid.org/0000-0002-7569-2414

${ }^{3}$ Este Programa se desarrolla en vinculación con el Proyecto de Investigación SECYT (FCS-UNC) "La política de seguridad en Córdoba: análisis de sus transformaciones como problema público desde la experiencia de distintos actores sociales, en especial los jóvenes de sectores populares en el período 2013 y 2017 ”. El equipo de trabajo estuvo integrado por: Valeria Plaza (directora), Susana Morales (co-directora), Magdalena Brocca, Natalia Danieli, Antonella Pestoni, Delia Sánchez y Agustina Zunino (investigadoras). Agradecemos también los aportes de los Drs. Natalia Bermúdez y Nicolás Cabrera del Núcleo de Antropología sobre Violencia, Muerte y Política, radicado en el IDACOR-CONICET, Museo de Antropología (FFyH-UNC). 
organizaciones sociales para el registro y sistematización de las prácticas de violencia institucional en el contexto de pandemia/ post pandemia.

Palabras clave: Fuerzas de Seguridad, ASPO, Violencia Institucional

\section{Abstract}

From the Extension Program "Security and Human Rights" of the Faculty of Social Sciences (FCS) of the National University of Córdoba (UNC) we carried out a survey of cases of abusive practices by the security forces concerning of arrests and controls established during the COVID pandemic in the Province of Córdoba.

We proposed to understand the conditions, situations and actors that enable them, as well as generate instances of transmission with social organizations and the university community, and articulation with state actors to contribute to the design of preventive policies.

In the present work, we then characterize the registration process carried out at the first moment of the pandemic, the actions and strategies of visibility and articulation undertaken and finally we propose some conceptual and methodological questions in relation to the strategies of joint work with social organizations for the registration. and systematization of institutional violence practices in the context of pandemic / post-pandemic.

Keywords: Security Forces, ASPO, Institutional Violence

\section{Introducción}

El objetivo de este escrito consiste, en primer lugar, en socializar las acciones que se realizaron desde la articulación entre investigación y extensión , en el marco desde el Programa de Extensión "Seguridad y Derechos Humanos" de la Facultad de Ciencias Sociales (FCS) de la Universidad Nacional de Córdoba (UNC) en contexto de pandemia; y en segundo lugar, nos proponemos realizar algunos planteos conceptuales y metodológicos en relación a las estrategias de registro y sistematización en relación a la violencia institucional.

Para ello, presentaremos de manera esquemática el contexto provincial en el que se inserta este relevamiento, los antecedentes extensionistas del equipo y las acciones realizadas en el marco y con posterioridad al relevamiento realizado a los fines de presentar la experiencia de manera integral y situada. Nos proponemos presentar esta experiencia como un aporte al debate en relación a las estrategias de registros de casos de violencia institucional tanto en el plano conceptual como metodológico. 


\section{Breve caracterización del contexto}

Sostenemos que en la Provincia de Córdoba, la política de seguridad provincial, luego de un proceso post-dictadura de disputa de campos policiales y no policiales (Hathazy, 2017), consolidó un desplazamiento de los segundos, constituyendo una arquitectura institucional y política caracterizada por la fuerte centralidad del componente policial en el período 2000/2015 (Brocca y otros, 2014; Carbajo, 2016). Esta centralidad policial además de generar consecuencias en el territorio urbano significó una sistemática oposición a las políticas nacionales de regulación de las fuerzas de seguridad tanto en lo discursivo como en medidas concretas. En el proceso electoral del 2015 ganó otra vez el oficialismo local, teniendo como eje de campaña el paradigma represivo en cuestiones de seguridad y la continuidad de las políticas implementadas. Sin embargo, una vez que asumió la actual gestión provincial (la segunda del Gobernador Schiaretti, iniciada en diciembre de ese año), propuso un discurso más inclusivo que su predecesor y se produjeron cambios normativos, institucionales y operativos, implementando medidas de política de seguridad preventiva como la policía barrial y los Consejos Barriales.

Estos cambios supusieron transformaciones tanto en la misma fuerza policial, en su dinámica operativa, como en su relación con los distintos actores sociales. Sin embargo, y como consecuencia del contexto sanitario, en la actualidad se suspendieron las reuniones mensuales de Consejos Barriales y la Policía Barrial se encuentra en reestructuración de funciones ya que, desde el inicio de la pandemia, colaboró en las tareas de control de la circulación, por lo que se suspendieron sus tareas preventivas en los barrios ${ }^{4}$.

Otro cambio significativo, fue el cambio normativo: se pasó de un Código de Faltas a un Código Contravencional con limitaciones a la potestad policial sobre todo el plano procedimental y esto trajo aparejado una reducción de las detenciones contravencionales. El Informe Anual de Gestión del Área Contravencional del Ministerio Público Fiscal del año 2016 señala un dato central: se declaran 8651 personas detenidas preventivamente por contravenciones, dando lugar a una estrepitosa caída de las detenciones contravencionales que se realizaban con el anterior Código de Faltas (que oscilaban entre 30 y 50.000 detenciones anuales ${ }^{5}$ ).

La disminución de las detenciones contravencionales no supone la disminución de otras prácticas abusivas y violentas en el espacio público, que quedan por fuera de

\footnotetext{
${ }^{4}$ Fuente: https://www.policiacordoba.gov.ar/Noticias/contenido/4727/reestructuracion-de-la-policiabarrial/

${ }^{5}$ En el informe anual se realiza una proyección anual de 12.888 presos contravencionales en toda la provincia de Córdoba, lo que implica una disminución del $82 \%$ de la cantidad de detenidos en relación a años anteriores con el Código de Faltas.
} 
todo registro y posibilidad de control (Sain 2008; Kessler y otros, 2016). Se presentan en el plano local los hostigamientos policiales que parecen no responder necesariamente a cambios normativos sino a políticas policiales y que han sido objeto de indagación en investigaciones académicas y en informes de Derechos Humanos en los últimos años en otros contextos provinciales (CELS, 2016).

En Córdoba, consideramos que las prácticas de hostigamiento policial se incrementaron. En las interacciones policiales donde no hay registro, donde al no aplicar la normativa vigente (El Código de Convivencia o el Código Penal), no hace falta acudir a una acción por parte de los destinatarios, a la comisión de un hecho tipificado, sino que se acude a otros elementos para "justificar" la interacción policial. Aparecen en los relatos de los trabajos de campo del equipo, la continuidad de determinadas prácticas de hostigamiento (golpes, cachetadas) y la aplicación de una gran dosis de discrecionalidad, permeadas por la violencia física, la vigilancia y el control de la policía sobre los jóvenes, bajo la óptica de la dominación territorial del Estado y la falta de registro de las mismas (Brocca y otros, 2014 y 2020, Plaza Schaefer, 2020). Esto además es presentado como un elemento desmovilizador y al tratarse de microagresiones, se presentan como más fácil de ser naturalizadas por los sujetos que las padecen cotidianamente y es por ello que se propone brindar herramientas a los jóvenes y esto se presenta como relevante en este punto: desde hace más de 12 años, son las organizaciones de jóvenes las que generaron los cuestionamientos más importantes a la política de seguridad, haciendo públicas las implicancias del control policial territorial que, sin embargo, siguen sin ser convocados como actores sociales en las nuevas medidas de la política de seguridad preventiva, como los Consejos Barriales (Plaza Schaefer, 2020).

En el plano carcelario, y como consecuencia de las políticas punitivistas, la persecución del narcomenudeo por la justicia provincial, la utilización de la prisión preventiva como regla y las limitaciones a las liberaciones anticipadas (libertad condicional y asistida) así como la baja utilización de las medidas alternativas a la prisión, la población carcelaria en Córdoba ha tenido un aumento sostenido en los últimos años. Las últimas cifras oficiales de que se dispone son de 2019. En ese momento había en la provincia de Córdoba 9962 personas privadas de la libertad (9466 varones, 495 mujeres y 1 trans) y se prevé que esta cifra siga aumentando. Cabe señalar que, del total de las personas privadas de la libertad en establecimientos penitenciarios, 5690 (57\%) tienen entre 18 y 34 años cifra que escala al 82,1\% (8178) si incluimos a las personas que tienen entre 35 y 44 años. Consideramos que existe una falta de registro oficial y escasas respuestas estatales ante las muertes bajo custodia en espacios de encierro. Particularmente en los últimos años en el EP3 de Bouwer (Establecimiento Penitenciario N³- Cárcel de Mujeres) se produjeron una serie de 
"suicidios" que fueron caratuladas como "muertes de etiología dudosa" que dieron lugar a una crisis penitenciaria y expusieron la falta de estrategias judiciales efectivas para la investigación y registro de las muertes en custodia.

En relación con la situación de lxs jóvenes privados de la libertad también es problemática. El Complejo Esperanza alberga a jóvenes varones en conflicto con la ley penal, en la actualidad son aproximadamente 140. El mismo está compuesto por 3 institutos y un centro de admisión: "Nuevo Sol” que alberga a jóvenes de 16 años en adelante (alrededor de 90 personas), "Horizonte" con unos 20 jóvenes (en general jóvenes vinculados a delitos contra la integridad física), y "San Jorge" donde residen jóvenes menores de 15 años (inimputables). Está ubicado en Camino a 60 cuadras en la localidad de Bouwer. Las jóvenes mujeres son alojadas en el Centro socioeducativo de Adolescentes Mujeres (CeSAM) ubicado en el antiguo edificio de Barrio Nueva Córdoba (fue trasladado allí durante el ASPO). La situación se agrava aún más en el caso de los niños y jóvenes alojados en el sector del régimen especial llamado de "resguardo" (confinamiento en solitario en una celda pequeña, sin luz, sin baño, ni agua durante 23 horas al día). La partida anual presupuestaria destinada a la Secretaría Nacional de Niñez, Adolescencia y Familia (SeNAF) es insuficiente: los y las jóvenes no tienen alimentos o elementos de higiene en el Complejo Esperanza. Los centros socioeducativos como el Complejo Esperanza y CeSAM, que alojan jóvenes en conflicto con la ley penal, también atraviesan condiciones gravísimas. A su vez, se produjeron traslados de presos adultos de la UCA (Unidad de Contención del Aprehendido) al Complejo Esperanza, vulnerando una vez más los derechos de las y los jóvenes y cediendo espacios propios como ya ocurrió con el Centro de Reorientación del Menor (CROM), actual UCA 1, violando todos los tratados internacionales y leyes nacionales respecto de con quiénes tienen que compartir lugar jóvenes y adolescentes en conflicto con la ley penal. Significa un proceso irreversible, es decir, la provincia no invierte en servicio penitenciario y a las consecuencias las pagan jóvenes de la SeNAF.

En relación con las Residencias de niños, niñas y jóvenes (NNA) con medidas de protección, que son las residencias que albergan a niñxs que se encuentran sin cuidados parentales, o transitaron por situaciones de vulneraciones que ponen en riesgo su integridad física y emocional (abusos, malos tratos, ausencia de alimentación y cuidados de salud, etc.), y para los cuáles se tomó "medida excepcional", si bien el régimen de alojamiento pareciera no constituir encierro de por sí, muchas de las características de regulaciones de la vida cotidiana se asemejan a las descriptas en relación al Complejo Esperanza. Además de infraestructuras edilicias deterioradas, inadecuadas y poco equipadas para que NNA realicen allí su vida cotidiana de manera digna, también el personal de la SeNAF advierte la 
constante falta de recursos alimentarios, vestimenta, atención de salud y acompañamiento en situaciones de padecimientos por consumo.

El encierro oculta vulneraciones que se producen al interior de las instituciones: solo pueden "salir" jóvenes y niñxs que asisten al sistema educativo (muchos no van a la escuela) y en muy escasas situaciones se gestionan incorporaciones a instituciones y organizaciones barriales (culturales, recreativas, deportivas).

Entonces, de los cambios normativos no hay ni un canal directoa las transformaciones en las prácticas y culturas institucionales. No se produce una ruptura necesaria del paradigma de la situación irregular al de protección de derechos, y es por ello para relevar la situación real de la niñez institucionalizada en general, de los jóvenes en conflicto con la ley penal, es necesario monitorear los cambios institucionales, sociales y legales asociados (Daroqui y Guemureman, 2001; Cesaroni 2010, Guemureman 2015, Beloff, 2016). Esto sería propio de "una visión normativa que lleva a olvidar que el derecho, para ser transformador, debe estar acompañado de diferentes grados de consenso social y de prácticas y acciones que lo modelen y le den vida" (Villalta \& Llobet, 2015; Medan, M.; Villalta, C. y Llobet, V. 2019). En efecto, los tratados internacionales como las nuevas legislaciones de los países, entre ellos Argentina que constitucionalmente consagra a la Convención de los Derechos del Niño, promueven la desjudicialización, especialización y excepcionalidad de la privación de la libertad las que, en caso de aplicarse, deberá ser del menor tiempo posible y revisarse periódicamente (Beloff, 2016), y lamentablemente, no es esto lo que ocurre en las prácticas institucionales.

Finalmente, en relación a la situación de vulneración de derechos se expresaron preocupaciones en las instituciones de salud mental. Se considera que justamente por no haber conflicto con la ley penal, en estos lugares de privación de la libertad por salud mental que responden a criterios sanitarios, las vulneraciones aparecen más invisibles. El Observatorio de Salud Mental y Derechos Humanos y la organización Confluir, usuarios en acción por el derecho a la salud mental, organizaciones de Córdoba que forman parte del Consejo Consultivo de Salud Mental presentaron informes detallados sobre la situación de la salud mental en Córdoba ${ }^{6}$.

Es importante poner de manifiesto la falta de información pública acerca de estas situaciones en contextos de encierro en general para dar cuenta de la importancia de generar registros. Consideramos que las políticas de seguridad, penitenciarias y en relación a los contextos de encierro en general se modificaron en el contexto de pandemia y se focalizaron en la apelación a las tareas de control

\footnotetext{
${ }^{6}$ Véase: http://observatoriosmyddhh.org/el-consejo-consultivo-declara-preocupacion-sobre-la-situacionde-la-salud-mental-en-cordoba/
} 
sanitario y restricción de circulación y es por ello es que nos propusimos intervenir en el relevamiento en un contexto particular y limitado de algunas de ellas.

\section{Antecedentes extensionistas}

El equipo viene trabajando de manera articulada y sostenida con organizaciones sociales y de derechos humanos desde hace un tiempo. Como antecedentes inmediatos, podemos remontar a agosto del 2018 momento en el que, como Equipo de Investigación, acompañamos la relación del Comité Nacional para la Prevención de la Tortura (CNPT) con las organizaciones de la sociedad civil locales. El CNPT es un organismo estatal independiente para la prevención de la tortura y los tratos o penas crueles, inhumanas o degradantes, creado por la ley nacional 26.827, en cumplimiento de lo dispuesto por el Protocolo Facultativo de la Convención Contra la Tortura de la ONU. Comenzó a funcionar en diciembre de 2017 y desde el año 2020, y en el marco de su mandato, realiza inspecciones periódicas no anunciadas a lugares donde se encuentren o puedan encontrarse personas privadas de libertad por orden judicial, administrativa o de cualquier otra autoridad en la Provincia de Córdoba. En una primera etapa, las organizaciones plantearon los establecimientos de encierro o detención que aparecieron como prioritarios para que el CNPT realizara las inspecciones; y se acompañó en la construcción de datos para los informes.

En ese proceso, se evidenciaron dos cuestiones: por un lado, la necesidad de las organizaciones de conocer los instrumentos nacionales e internacionales de recomendaciones a la hora de elaborar registros de casos y de sistematización de información y, por otro lado, la dificultad de visibilizar las prácticas de hostigamiento policial que se producen, sobre todo, en las calles o lugares públicos de la ciudad (plazas, esquinas, ingresos y egresos de barrios, etc.).

Luego de esto, presentamos un seminario de extensión durante el 2019 y generamos un espacio de intercambio de conocimientos, saberes y experiencias en torno a la violencia institucional en Córdoba. Desde el equipo docente nos propusimos brindar herramientas conceptuales y jurídicas para la intervención en las vulneraciones de derechos en el campo de la violencia institucional y aportar a la generación de herramientas de registro y sistematización de estas.

Los talleres estuvieron dirigidos, por un lado, (A) a organizaciones sociales y referentes territoriales (culturales, institucionales y políticos); y por otro, (B) se convocó a jóvenes organizados que desarrollan sus actividades en distintas zonas de la ciudad. A lo largo de los módulos se mantuvo una participación aproximada de 30/40 asistentes. 
Los módulos que compusieron esta propuesta se plantearon como instancias de formación y debate en relación a las vulneraciones de derecho en el área de la violencia institucional en Córdoba, partiendo de situarlas en el marco de procesos de transformaciones de la política pública provincial y su articulación con el marco normativo internacional y sus organismos. Estuvieron orientados a brindar herramientas conceptuales y jurídicas que permitan la construcción y circulación del conocimiento con las organizaciones sociales participantes, procurando avanzar en el diseño de instrumentos de registros, construcción de indicadores locales, a la planificación de acciones conjuntas y la importancia del trabajo en red.

A lo largo del seminario, se sistematizaron en perspectiva histórica (línea de tiempo) las estrategias emprendidas por las organizaciones sociales en la provincia de Córdoba, analizando los alcances y limitaciones de cada una de ellas de la última década y, por otra parte, se presentaron posibles diseños de protocolos para el registro por parte de las organizaciones sociales en la política de seguridad y penitenciaria.

\section{Las estrategias de articulación conjunta y el relevamiento en pandemia}

Durante el 2020, en función del contexto sanitario, se realizaron reuniones virtuales con organizaciones sociales de la provincia de Córdoba que trabajan en torno a la problemática de la seguridad y derechos humanos. La mayoría de estas organizaciones están nucleadas en la Comisión "Seguridad y Derechos Humanos" del Consejo Social de la FCS ${ }^{7}$.

En función de demanda de las organizaciones con quienes veníamos fortaleciendo la articulación en el seminario antes reseñado, nos propusimos un relevamiento de casos sobre prácticas abusivas por parte de las fuerzas de seguridad en el marco de las detenciones y control establecidos para garantizar el aislamiento social y obligatorio en Provincia de Córdoba. Además, se pretendió comprender las condiciones, situaciones y actores que habilitan las mismas.

\section{a) La herramienta y la metodología}

La metodología consistió en un formulario on-line destinado a informantes clave, como organizaciones sociales y a personas que tomaban conocimiento de casos. El acceso al formulario fue facilitado por páginas de la UNC y medios de

\footnotetext{
7 La poderosa, Mesa de DDHH Córdoba-Comisión Violencia Institucional, Confluir, Familiares de Desaparecidos y Detenidos por Razones Políticas Córdoba, Asociación Pensamiento Penal Córdoba, Justicia Legítima Córdoba, Sub-Comisión de Derechos Humanos del Sindicato de Luz y Fuerza de Córdoba, Fundación UMEP, Org. Indígena EPIKAMBA, SEHAS, Observatorio de la situación de salud, socioambiental y económica de niños, niñas y adolescentes (Sierras Chicas).
} 
comunicación. En virtud del vínculo construido con referentes de organizaciones territoriales, se logró un aporte de casos que podrían haber sido invisibilizados dadas las limitaciones de un formulario on-line en cuanto a accesibilidad. Se registraron más de 30 casos durante los meses de abril a junio del 2020, no sólo de Capital, sino también de localidades del interior.

El cuestionario on-line se focalizaba sólo en detenciones policiales caratuladas como violaciones a las normativas de la ASPO. Allí, se permitía diferenciar entre: abuso y arbitrariedad en el uso de la violencia policial, hostigamiento y/o maltrato físico en el momento de la detención, hostigamiento y/o maltrato verbal en el momento de la detención, falta o información errónea sobre la modalidad y condiciones de la detención (a donde iba ser alojado, cuanto tiempo) y/o amenazas sobre detenciones futuras. Además, se solicitaba narrar brevemente el hecho, aclarando la zona en donde ocurrió. En primera instancia, los casos recabados sirvieron para dar a conocer mediante un informe preliminar, las prácticas policiales durante los primeros meses del ASPO. Luego de decretado el distanciamiento social, se continuó con actividades de visibilización por parte del equipo.

b) Fuerzas de Seguridad y COVID: ¿cómo respondieron las agencias de fuerza policial a la Pandemia?

Consideramos que la policía produjo numerosas interacciones violentas que podrían haberse evitado, si la información se hubiera difundido de manera complementaria con otros actores estatales - muy ausentes en la primera etapa de la pandemia- y se hubiera articulado con actores sociales y comunitarios.

A su vez, las medidas arbitrarias se profundizaron en sectores populares y se extendieron a barrios de clase media. Entonces, las prácticas de hostigamiento policial no solo se profundizaron en los sectores "objeto" tradicional de estas interacciones arbitrarias y violentas, sino que se extendieron a otros sectores sociales, quienes se mostraron más preocupados por la presencia de las fuerzas de seguridad.

Se puede afirmar que la política social de fuerza de seguridad en Córdoba presenta problemas estructurales severos ${ }^{8}$, lo cual es subrayar una obviedad.

\footnotetext{
${ }^{8}$ Se reconoce como antecedentes inmediatos las investigaciones realizadas por este equipo desde el año 2013 para distintos organismos públicos (Brocca, y otros, 2014; Brocca y otros, 2015, Bologna, Gómez, Morales y Plaza, 2017)), las investigaciones realizadas para SECYT para las convocatorias 2016/2017 y 2018/2019 y distintas tesis individuales vinculadas a las políticas de seguridad en Córdoba (Plaza Schaefer, 2016), su relación con procesos de segregación residencial, sobre las implicancias de la interacción policial con los jóvenes cordobeses (Bologna y otros, 2017; Plaza Schaefer, 2018 a, b y c; Brocca, 2019; Pestoni, Danieli y Sánchez, 2019), así como trabajos vinculados a los aspectos subjetivos de la seguridad en nuestra provincia (Morales, 2016) y las modificaciones en el marco normativo de la política de seguridad (Danieli y Pestoni, 2019 a). En estos trabajos, además de sistematizar las características centrales y las transformaciones de las políticas de seguridad en nuestra provincia, se fueron construyendo indicadores sobre uso de la fuerza (Morales, Brocca y
} 
Evidentemente, la pandemia de COVID-19 ha venido profundizar al extremo aquellas falencias.

Este tipo de políticas de seguridad se presentan enunciadas como grandes soluciones que se caracterizan por un claro exceso de significantes, con proyectos muy disímiles de violencia institucional y vulneración de derechos. Los conceptos se vacían de sentido y de allí el exceso de significantes: la razón instrumental (el cómo) domina el campo (la lógica de soluciones domina la lógica de problemas). Las grandes soluciones logran una clausura simbólica, en la que se impide la problematización de la situación que viven los y las ciudadanos, la cual al naturalizarse obtura la posibilidad de su transformación. Y entonces se pasa a depender de los efectos de las políticas concebidas como universales y que, por lo tanto, desconocen las singularidades. Se configura así una lógica de políticas sin sujetos, o sujetos devenidos objetos para esas políticas que fueron estructuradas, basadas en una racionalidad extraña a esas singularidades, y esas políticas suelen provenir de enlatados fabricados en serie por los organismos internacionales.

Consideramos así que las medidas arbitrarias se profundizaron en sectores populares y se extendieron a barrios de clase media. De este modo, las prácticas de hostigamiento policial no solo se profundizaron en los sectores "objeto" tradicional de estas interacciones arbitrarias y violentas, sino que se extendieron a otros sectores sociales, quienes se mostraron más preocupados por la presencia de las fuerzas de seguridad.

En las situaciones donde se presentó una profundización del hostigamiento en sectores tradicionales, se encuadran principalmente prácticas de malos tratos físicos (golpes) y verbales, y prácticas de hostigamiento que generan vulneraciones de derechos humanos que incrementan la situación de exclusión y precariedad de estos sectores. En este primer grupo, se incluyen las prácticas de hostigamiento a trabajadorxs sexuales. En el segundo grupo, consolidado por poblaciones no acostumbradas a estas interacciones policiales, aparece con frecuencia que el hostigamiento o arbitrariedad tiene una fuente o habilitación legal (la restricción de la circulación sin documentación correspondiente) pero por determinadas circunstancias, la situación se convierte en hostigamiento.

Además de buscar visibilización de estas situaciones y realizar informes destinados a instituciones gubernamentales, se planteó la necesidad de obtener un protocolo claro de intervención por parte de las fuerzas de seguridad.

Plaza, 2018) y se dio cuenta de un conjunto de procesos ligados a la ampliación de los ámbitos de la vida cotidiana que fueron atravesados por una creciente policiación como lógica central de resolución de conflictos. 
A lo largo del recorrido teórico y práctico de nuestro equipo de investigación, estaba previsto que este contexto iba a generar características particulares de interacción, lo cual permitió accionar rápidamente estrategias para evaluarlas.

En primer lugar, se considera que si bien el tema de la violencia institucional contó con una publicidad importante como tema durante el ASPO, creemos que a través de esta herramienta se logró un aporte de casos que podrían haber sido invisibilizados por falta de cobertura mediática.

c- ¿Qué se hizo con los datos?

A partir de los datos recabados, se realizaron articulaciones con distintas organizaciones y se realizaron las siguientes acciones:

- Conversatorio (julio 2020):

En primer lugar, se realizó un conversatorio en el que junto con equipos de investigación de otras provincias y organismos de DDHH como el CELS, se abordaron los resultados del relevamiento y se analizaron otros contextos provinciales (Salta y Santiago del Estero, que habían producido relevamientos similares), se propusieron hipótesis y en términos de proyección se planteó la necesidad de articulación entre provincias (sobre todo en relación a la producción de datos y construcción de diagnósticos locales coordinados).

- Presentación virtual a autoridades locales y nacionales del área de Seguridad y Derechos Humanos (julio 2020):

En torno a los resultados del relevamiento descrito en el punto anterior se realizó una presentación institucional ante autoridades locales y nacionales. En la reunión virtual participaron el Sr. Secretario de Derechos Humanos de la Nación, Horacio Pietragalla; Sr. Secretario ejecutivo del Consejo Federal de Derechos Humanos, Agustín Di Toffino; Sra. Subsecretaria de Programación Federal y Articulación Legislativa, Silvia La Ruffa; Sr. Subsecretario de Control y Transparencia Institucional, Esteban Rosa Alves; Srta. Subsecretaria de Bienestar, Derechos y Políticas de Género, Sabrina Calandrón del Ministerio de Seguridad de la Nación; Sr. Director del Observatorio de Derechos Humanos del Senado de la Nación, Juan Martín Fresneda; Sr. Secretario de Derechos Humanos de la Provincia de Córdoba, Calixto Angulo y el Sr. Secretario de Derechos Humanos de la Municipalidad de Córdoba, Emiliano Fessia. En la presentación, se propusieron además ejes posibles de articulación en relación a la temática. 
- Nota firmada por las organizaciones locales y el CELS al Gobernador de la Provincia de Córdoba (setiembre 2020):

En setiembre del 2020, se produjo la remoción de parte de la cúpula policial de la capital luego del asesinato del adolescente Valentino Blas Correa, de 17 años, y de los intentos por encubrir el hecho, y en ese marco, en articulación con las organizaciones sociales con quienes se venía trabajando, se redactó una nota para transmitirle nuestra preocupación por los graves hechos de violencia institucional protagonizados por la Policía de Córdoba en los últimos meses y solicitar información sobre la adopción de medidas de fondo orientadas a controlar y profesionalizar esta fuerza policial. Esta nota fue firmada por numerosas organizaciones sociales locales, la FCS-UNC y el Centro de Estudios Legales y Sociales (CELS). Esta misiva fue contestada por el Sr. Ministro de Seguridad (octubre del 2020) quien enumeró las medidas normativas y ejecutivas implementadas y se anunciaban cambios en las políticas del sector 9 .

- Informe integral sobre las violencias policiales y las vulneraciones de derechos humanos en contextos de encierro, firmado por las mismas organizaciones (diciembre 2020):

Luego de aquella respuesta, se realizó una nueva reunión virtual con las organizaciones sociales firmantes ( 6 de noviembre) en donde se evaluaron las acciones a seguir, considerando la respuesta emitida desde el Ministerio de Seguridad y se propuso la elaboración de un diagnóstico participativo no sólo en torno a la violencia policial sino también en relación a las violencias y vulneraciones de derechos en contextos de encierro - tanto penitenciarios y del sistema penal juvenil, como de salud mental. La reunión virtual se realizó con la presencia de más de 15 organizaciones sociales y espacios de investigación y extensión de distintas facultades de la UNC que trabajan en relación a la temática ${ }^{10}$. En la reunión se propuso redactar

\footnotetext{
${ }^{9}$ Las organizaciones sociales con las que se trabajó fueron aquellas nucleadas en la Comisión "Seguridad y Derechos Humanos" del Consejo Social de la Facultad de Ciencias Sociales de la UNC que coordino en el marco del Programa de Extensión "Seguridad y Derechos Humanos" de dicha facultad. Las organizaciones son: La poderosa, Mesa de DDHH Córdoba-Comisión Violencia Institucional, Confluir, Familiares de Desaparecidos y Detenidos por Razones Políticas Córdoba, Asociación Pensamiento Penal Córdoba, Justicia Legítima Córdoba, Sub-Comisión de Derechos Humanos del Sindicato de Luz y Fuerza de Córdoba, Fundación UMEP, Org. Indígena EPI-KAMBA, SEHAS, Observatorio de la situación de salud, socioambiental y económica de niños, niñas y adolescentes (Sierras Chicas).

${ }^{10}$ Las organizaciones firmantes y que participaron de las reuniones virtuales fueron: La poderosa, Mesa de DDHH Córdoba, Familiares de Desaparecidos y Detenidos por Razones Políticas Córdoba, Asociación Pensamiento Penal- Capitulo Córdoba, Secretaría de Derechos Humanos del Sindicato de Luz y Fuerza de Córdoba, Fundación Un Mundo Mejor es Posible (UMMEP), Org. Indígena EPI-KAMBA, Justicia Legítima
} 
un informe que diera cuenta de un diagnóstico local de las situaciones de las cárceles e instituciones de salud mental y la violencia policial desde el inicio de la pandemia. Así se elaboró un diagnóstico para ser presentado ante autoridades locales del poder ejecutivo y legislativo provincial, y en el mismo informe se elaboró un análisis de las medidas implementadas con posterioridad al homicidio de Blas Correa: un protocolo de uso de armas de fuego, y dos proyectos legislativos: la modificación de la ley de seguridad y la modificación del Tribunal de Conducta Policial. Este informe se presentó a finales de diciembre.

d) Vinculación territorial fuerzas de seguridad con Jóvenes en ASPO

Ante esta situación de aislamiento social, preventivo y obligatorio cabe preguntarse ¿qué pasó en el vínculo jóvenes-policía? La política de seguridad de Córdoba se caracteriza históricamente por un despliegue de la fuerza represiva del Estado en términos de defensa social.

A nivel provincial, las políticas de seguridad estuvieron centradas en el ejercicio de poder sobre los sectores vulnerables y no vulnerables, lo cual trajo como consecuencia la construcción como enemigos a los jóvenes pertenecientes a los sectores populares, los mismos que desde la aplicación de las políticas neoliberales fueron expulsados del sistema económico. Esta acción, domestíca las relaciones sociales, "civiliza" las costumbres públicas y privadas, construye "formas de habitar cotidianas y contemporáneas" (Crisafulli, 2015).

Se recabaron dos casos de jóvenes que tuvieron gran revuelo mediático, como ser el caso de Valentino Blas Correa ${ }^{11}$ ( el joven de 17 años muerto por disparos de personal policial) que, por la forma y el lugar donde el hecho tuvo lugar, generó una importante reacción social y por ello, una inmediata visibilidad mediática y social .

El asesinato de Valentino es, además de terrible en sí mismo, un caso que cristaliza y evidencia un modo de violencia policial que viene implementándose hace tiempo para con sectores más vulnerables. Otro aspecto gravísimo es el intento de encubrimiento y todas las maniobras alrededor para intentar una justificación

Córdoba, SEHAS (Servicio Habitacional y de Acción Social), Colectivo cordobés por los derechos de niños niñas y jóvenes, VillaNos Radio 100.7 FM - Villa Carlos Paz. También participaron los siguientes equipos de investigación y extensión de la UNC: Núcleo de Estudios e Intervención en Seguridad Democrática (NEISeD). Facultad de Filosofía y Humanidades, la Cátedra de "Psicología Social y Vida Cotidiana" de la Facultad de Ciencias Sociales y Cátedra de Psicología Social Facultad de Psicología, Proyecto de Investigación SECYT "Jóvenes en situación de desigualdad social: aproximaciones desde el enfoque biográfico a la relación vida y muerte"-Facultad de Psicología, Proyecto de investigación CIECS- FCS UNC "Biosubjetividades: Neoliberalismo, Control y Resistencias", Proyecto SECYT "Producción de subjetividad y acceso a derechos. Sentidos en torno al Programa Universitario en la Cárcel”, Grupo Derecho y Control, CIJS UE-CONICETFacultad de Derecho.

${ }^{11}$ https://www.lanacion.com.ar/seguridad/gatillo-facil-cordoba-mama-victima-sr-fiscal-nid2456844 
imposible sobre el accionar policial violento. No se trata de hechos aislados ni de prácticas nuevas, son gestos que trazan un esquema de impunidad, de creer que tienen la potestad de actuar con la más extrema violencia sin consecuencias graves. Ahora, sí es significativo que se amplíe el espectro de la sociedad al que se le aplican. El contexto de la pandemia y la dotación a las fuerzas policiales de mayores prerrogativas intensificaron seguramente los abusos por parte de estas fuerzas. Sin embargo, debemos preguntarnos por qué es que recién cuando esta violencia se ejerce contra alguien de clase media, la sociedad y los medios reaccionan, y hasta se producen ciertos gestos políticos, como el cambio de la cúpula policial.

Se puede mencionar el último hecho del accionar violento de la policía de la provincia de Córdoba que terminó asesinando a Joaquín Paredes ${ }^{12}$ de 15 años, en la localidad de Paso Viejo en el noroeste provincial. En la madrugada del domingo 25 de octubre, un grupo de jóvenes del pueblo se encontraba en la plaza de Paso Viejo, cuando fueron atacados violentamente por un grupo de policía que, sin mediar palabra, empezó a disparar llevándose la vida de Joaquín e hiriendo a dos jóvenes más. El gobierno de la provincia de Córdoba, recién luego de estos de hechos elaboró un protocolo de actuación.

Este caso configura un modo de entender la seguridad, un modo de considerar a la población joven como sospechosa. Todos los pasos, nos conducen a sostener que existen prácticas muy consolidadas, una forma de actuar que supera toda la preparación y los protocolos de actuación de los uniformados en la calle. Esta manera de ejercer la autoridad demuestra que algunas de las divisiones de la policía de Córdoba trabajan autónomamente, y que existe un método de despliegue en el territorio selectivo de prácticas abusivas, ilegales de aplicación de la fuerza física y malos tratos.

Esto no son acciones aisladas. Vivimos todos los días en nuestros pueblos y ciudades el hostigamiento y persecución permanente de la policía de Córdoba contra los jóvenes de los sectores populares. Por eso mismo, demandamos la discusión urgente de un modelo de seguridad pública basado en valores democráticos, inclusivos y solidarios.

\section{Conclusiones}

Luego del proceso iniciado en conjunto con las organizaciones en relación a la sistematización de estrategias y registro de la violencia institucional con el seminario extensionistas, nos vimos obligadas a modificar la metodología y las actividades

\footnotetext{
${ }^{12}$ Véase: https://www.lavoz.com.ar/sucesos/ultimo-adios-a-joaquin-paredes-en-paso-viejo-desgarradorpedido-de-justicia-por-parte-de-fam
} 
propuestas en función de la no circulación de la pandemia y la consecuente imposibilidad de continuar las capacitaciones en los territorios donde se desarrollan.

Consideramos que, en ese contexto, hemos fortalecido el vínculo con algunas de las organizaciones sociales y hemos desarrollado una intensa actividad de vínculos institucionales, pero también de intervenciones mediáticas. Entendemos que intervenir a partir de información producida de manera sistemática es un aporte al debate público. Sin embargo, consideramos que es necesario aportar herramientas para que tanto la comunidad en general como en las organizaciones puedan reconocer las especificidades de la violencia institucional.

En los registros de las estrategias emprendidas que realizamos en el seminario, identificamos que nuestra provincia cuenta con una larga tradición en relación en visibilizar la cuestión, de la que las organizaciones sociales y de jóvenes han sido protagonistas. Sin embargo, faltan herramientas a la hora de registrar y sistematizar unas prácticas que se van modificando tanto por los contextos como por las implicancias que tienen las transformaciones normativas y operativas en las fuerzas de seguridad.

En el proceso transcurrido, se enunciaron posibles líneas de trabajo entre organizaciones sociales, la facultad y los distintos niveles del Estado con quienes se plantearon articulaciones. Sin embargo, muchas de ellas no prosperaron en este contexto particular de pandemia, por lo que nos proponemos en esta etapa, fortalecer las articulaciones con las organizaciones sociales y las estrategias de registro, y en función de ello, nos planteamos las siguientes inquietudes/ interrogantes:

- Revisar las limitaciones de la estrategia: el cuestionario online requiere de un conjunto de competencias específicas, así como accesibilidad tecnológica que no todas las organizaciones tienen. La mayoría de los casos registrados provinieron de la carga realizada o al menos difundida por alguna organización (salvo los casos relacionados con barrios de clase media y/o sectores universitarios que llenaron de manera individual el formulario por haberse enterado del mismo por difusión interna). Nos preguntamos entonces de qué manera se puede masificar una herramienta de estas características, ya que consideramos que en aquellos territorios de la provincia donde no hay organizaciones, no se registraron casos (no porque no hubo, sino porque no llegó la información del relevamiento).

- Ampliar el registro de casos: en el relevamiento realizado sólo registramos casos de violencia policial en pandemia. Consideramos que 
es posible ampliar ese registro a casos de violencia institucional más amplio, es decir, relevar los casos en cárceles y contextos de encierro en general (NNA en conflictos con la ley penal, instituciones de salud mental). Esto se debe a que las organizaciones con las que articulamos trabajan en estos contextos y, por lo tanto, demandaron la sistematización de estas prácticas. Esta situación nos generó la necesidad de profundizar el concepto de violencia institucional, identificando que abarca y que no.

- Proponer actualizaciones de los registros: identificamos que las prácticas relevadas están insertas en procesos dinámicos, no sólo en relación con los lugares donde se desarrollan, sino que se modifican también y en períodos cortos de tiempo, en relación a modificaciones de política pública en general (por ejemplo, en función de la cuestión sanitaria: detenciones o multas, cordones sanitarios u operativos barriales) y/o política policial. Por ello nos proponemos diseñar estrategias de monitoreo en conjunto con las organizaciones sociales.

La sistematización realizada en este trabajo tiene como finalidad recuperar una experiencia local, de articulación de espacios universitarios con organizaciones sociales no como una experiencia acabada sino en proceso, que además sufrió vaivenes en función del contexto de pandemia.

Nos propusimos compartir los interrogantes que nos surgieron en función de la continuidad en este contexto actual, para enriquecer la mirada en función de otras experiencias y, además, y en virtud de que existe un proyecto de ley en tratamiento sobre el registro de estos casos, visibilizar las complejidades del registro y la necesidad de construcción de herramientas consensuadas que puedan contemplar la heterogeneidad de prácticas que se suceden y que ya se están registrando. Es necesario aportar herramientas para que tanto la comunidad en general como las organizaciones puedan reconocer las especificidades de la violencia institucional.

Algunas cuestiones necesarias, en este sentido son diseñar organismos de control externos y con capacidad real de investigar la actividad policial, producir información estadística y cualitativa, sistematizada y de acceso público sobre el funcionamiento de las policías (lo que incluye, pero excede a las estadísticas sobre delitos), desarrollar protocolos de actuación con criterios claros de intervención policial y revisar los planes de formación y capacitación. Es importante considerar que la policía "no tiene formación para advertir de forma no violenta o amenazante sobre las normas" (Relevamiento COVID MINCYT-CONICET, 2020 p. 14) y por 
ello es necesario que esta información se difunda sobre todo por otros sectores estatales y se articule con actores sociales y comunitarios.

Además, es necesario desarrollar y pensar políticas en clave intersectorial (Subirats 2010 y Cunill-Grau 2012), evitando que sea uno de los sectores, el que acabe coordinando al resto. La intersectorialidad, desde esta última perspectiva, implica que diversos sectores gubernamentales no sólo entreguen a un mismo público específico los servicios que son propios de cada uno, sino que de manera articulada atiendan necesidades sociales o prevengan problemas que tienen complejas, diversas y relacionadas causas en sus orígenes.

El supuesto es que, si aspiramos a lograr, por ejemplo, el desarrollo integral de derechos humanos no basta con que cada sector (seguridad, salud, educación, SeNAF, género, etc.) haga lo que le corresponde de acuerdo con sus respectivos mandatos, ni siquiera significa evitar que se produzcan redundancias entre ellos. Implica que los sectores "se pongan de acuerdo" para actuar "conjuntamente" a fin de lograr un cambio social respecto de la situación inicial. Entonces, la política pública de seguridad desde un enfoque de derechos humanos, y no como política criminal. Esto implica que la Política Pública de Seguridad tenga, desde su concepción, un enfoque intersectorial para abarcar la problemática social desde la multiplicidad de factores.

\section{Referencias}

Brocca, M.; Morales, S.; Plaza Schaefer, V.; Crisafulli, L. (2014): "Policía, Seguridad y Código de Faltas”, en Mirar tras los muros: situación de los derechos humanos de las personas privadas de libertad en Córdoba. Ed. Universidad Nacional de Córdoba y Universidad Nacional de Río Cuarto, Córdoba, 427- 480.

Brocca, M.; Morales, S.; Plaza Schaefer (2020), “La seguridad en cuestión. Transformaciones en la cuestión securitaria como problema público. Córdoba, 2015 2017’, Revista DÍKÊ. Revista de Investigación en Derecho, Criminología y Consultoría Jurídica, Nro 28, octubre 2020- marzo 2021, Benemérita Universidad Autónoma de Puebla, pp 145-169

Beloff, M. (2016). ¿Qué hacer con la justicia juvenil?. Buenos Aires. Ad-hoc.

Carbajo, M.(2016): La educación policial y el gobierno de la seguridad en la provincia de Córdoba. Reformas en la formación policial, policialización de las políticas de seguridad y modelo tradicional de policía (2003-2013). Tesis Magíster en Ciencias Sociales Fac. CC. SS, UNC, Córdoba, Inédita.

CELS (2016). Hostigados. Violencia y arbitrariedad policial en los barrios populares. Informe financiado por la Unión Europea, 2016. 
Cesaroni, C. (2010). La vida como castigo. Los casos de adolescentes condenados a prisión perpetua en la Argentina. Grupo Editorial Norma: Buenos Aires.

Crisafulli, L. (2015). El camello y la galaxia contravencional. Reflexiones sobre el código de faltas en Córdoba, Argentina. Revista Crítica Penal y Poder(8), pp. 117.

Cunill-Grau, N. (2012). La intersectorialidad en las nuevas políticas sociales. Un acercamiento analítico-conceptual. Revista Scielo. Pp1-42.

Guemureman, S. \& Daroqui A. (2001). La niñez ajusticiada. Buenos Aires: Del Puerto.

Guemureman, S. (2015). Las políticas de seguridad dirigidas hacia adolescentes y jóvenes: planes, programas, y acciones tendientes al gobierno y gestión de la juventud vulnerable. En Guemureman, S. (Comp.). Politicas Penales y de Seguridad dirigidas hacia adolescentes y jóvenes. Buenos Aires: Editorial RubinzalCulzoni.

Hathazy, P. (2017) Juego político nacional y políticas provinciales: un análisis multiescalar de la emergencia y reconfiguración de la política de seguridad en Córdoba, Argentina (1984-1999), en prensa.

Kessler, G.; Zavaleta, A.; Alvarado, A. Y Zaverucha, J. (2016). Una aproximación a las relaciones entre policías y jóvenes en América Latina. Política y gobierno. Volumen XXIII • Número 1, pp. 201-229. Santiago de Chile.

Medan, M.; Villalta, C. y Llobet, V. (2019). Entre inercias burocráticas y evaluaciones sobre las familias: adolescentes privados de libertad en Buenos Aires, Argentina. Estudios Socio-Jurídicos, 21(1), 293-326. Doi: http://dx.doi.org/10.12804/revistas.urosario.edu.co/sociojuridicos/a.6309.

Plaza Schaefer, V. (2020). "De las detenciones contravencionales a las prácticas de hostigamiento policial. Reflexiones sobre los cambios y permanencias en los estereotipos de conflictividad policial desde la mirada de jóvenes organizados en la Ciudad de Córdoba”. Revista Delito y Sociedad. Revista de ciencias Sociales, No 49, enero-junio 2020 Programa de Estudios del Control Social, Universidad de Buenos Aires Programa Delito y Sociedad, Universidad Nacional del Litoral, pp. 129-154. DOI: https://doi.org/10.14409/dys.2020.49.e0006

Villalta, C. y Llobet, V. (2015). Resignificando la protección. Nuevas normativas y circuitos en el campo de las políticas y los dispositivos jurídico-burocráticos destinados a la infancia en Argentina. Revista Latinoamericana de Ciencias Sociales, Niñez y Juventud, 13, 167-180.

Relevamiento COVID MINCYT-

CONICET(2020)

https://www.conicet.gov.ar/wp-

content/uploads/Resumen_Ejecutivo_Covid-Cs.Sociales.pdf 
Sain, M. (2008). EL Leviatán Azul. Política y Policía en la Argentina. Buenos Aires: Siglo XXI.

Subirats, J. H (2010) “¿Tenemos las políticas públicas que necesitamos? Gobernanza y factores de cambio en la política y en las políticas". En El Estado y las Politicas Públicas en América Latina / Paula Amaya y otros... [et.al.]. - 1a ed. - La Plata: Universitaria de La Plata. 